\title{
Tumor Embolism Presenting as Rapidly-forming Cavitary Lesion
}

\author{
Joseph R. Sweigart ${ }^{\mathrm{a}}$, Brian Wolfe ${ }^{\mathrm{a}}$, Rondle Moubry ${ }^{\mathrm{b}}$, \\ Daniel T. Merrick ${ }^{\mathrm{c}}$, Edward D. Chan ${ }^{\mathrm{d}, \mathrm{e}, \mathrm{f}, \mathrm{g}, \mathrm{h}}$
}

\begin{abstract}
Cavitary lung lesions are most often associated with infectious processes, but cavities can develop rapidly in the setting of pulmonary infarction due to venous thromboemboli or malignant necrosis most comonly due to squamous cell carcinoma. Venous tumor embolism occurs when tumor cells invade the vasculature and then disseminate. Initial symptoms of tumor emboli to the lungs are typically similar to those of non-malignant pulmonary thromboembolism including dyspnea, hypoxemia, and normal or minimally abnormal chest radiography, but may also include progressive pulmonary hypertension and cor pulmonale. Definitive diagnosis can be made via lung tissue biopsy or demonstration of malignant cells from blood samples aspirated from the distal port of a wedged pulmonary artery catheter. We report the case of a patient who developed rapidly-developing, large lung cavities due to diffuse tumor emboli. A 64 year-old man with a history of lung adenocarcinoma
\end{abstract}

Manuscript accepted for publication September 9, 2011

${ }^{\mathrm{a}}$ Department of Medicine, University of Colorado Denver Anschutz Medical Campus, Aurora, Colorado, USA

${ }^{b}$ Department of Radiology, Denver Veterans Affairs Medical Center, Denver, Colorado, USA

${ }^{c}$ Department of Pathology, Denver Veterans Affairs Medical Center, Denver, Colorado, USA

${ }^{\mathrm{d} D i v i s i o n}$ of Pulmonary Sciences and Critical Care Medicine,

University of Colorado Denver Anschutz Medical Campus, Aurora, Colorado, USA

${ }^{e}$ Respiratory Section, Denver Veterans Affairs Medical Center, Denver, Colorado, USA

${ }^{\mathrm{f}}$ Department of Medicine, National Jewish Health, Denver, Colorado, USA

${ }^{\text {g}}$ Program in Cell Biology, National Jewish Health, Denver, Colorado, USA

${ }^{\mathrm{h}}$ Corresponding author: Edward D. Chan, D509, Neustadt Building, National Jewish Health, 1400 Jackson Street, Denver, CO 80206.

Email: chane@njhealth.org

doi:10.4021/jmc263w previously treated with lobectomy and chemotherapy was admitted with respitatory distress. He was tachycardic and hypoxic on presentation. Imaging studies revealed a small pulmonary embolus within the pulmonary artery supplying the left upper lobe as well as two large right-sided pulmonary cavities which were not present on outpatient imaging just one week prior. Laboratory studies revealed leukocytosis and abnormal liver enzymes but no definitive evidence of infectious or autoimmune pathology. Despite treatment with systemic anticoagulation and broad-spectrum antibiotics, he developed progressive respiratory failure as well as neurologically-devastating cerebral ischemic events. After discussion with his family, he was transitioned to comfort care and died shortly thereafter. Post-mortem examination revealed widespread occlusion of pulmonary vessels with malignant cells, including the vessels supplying the areas of the lung containing the cavities. Histopathologic staining was consistent with adenocarcinoma. Non-malignant thromboemboli were not found on gross or microscopic examination of his lung vasculature. He also had neoplastic involvement of the vessels in his splanchnic circulation. We believe that widespread tumor emboli caused his pulmonary cavities, respiratory failure, and neurologic deterioration.

Keywords: Tumor embolism; Cavitary lung lesion

\section{Introduction}

Tumor embolism (TE) occurs when neoplastic cells migrate into the vascular supply and then travel to distant areas of the body. Cancer cells likely have characteristics that enable them to deposit within microvasculature systems because neoplastic spread is thought to most frequently occur via the hematogenous route and the lung is the most common site for metastases [1].

Initial symptoms of TE to the lungs are often similar to those of non-malignant pulmonary venous thromboembolism (VTE) including shortness of breath, varying degrees of hypoxemia, and normal or minimally abnormal chest radiography [1]. Involvement of a significant portion of the pulmonary microvasculature can result in progressive pulmonary hypertension and cor pulmonale, although the classic signs of right heart failure may be seen in only a minority 

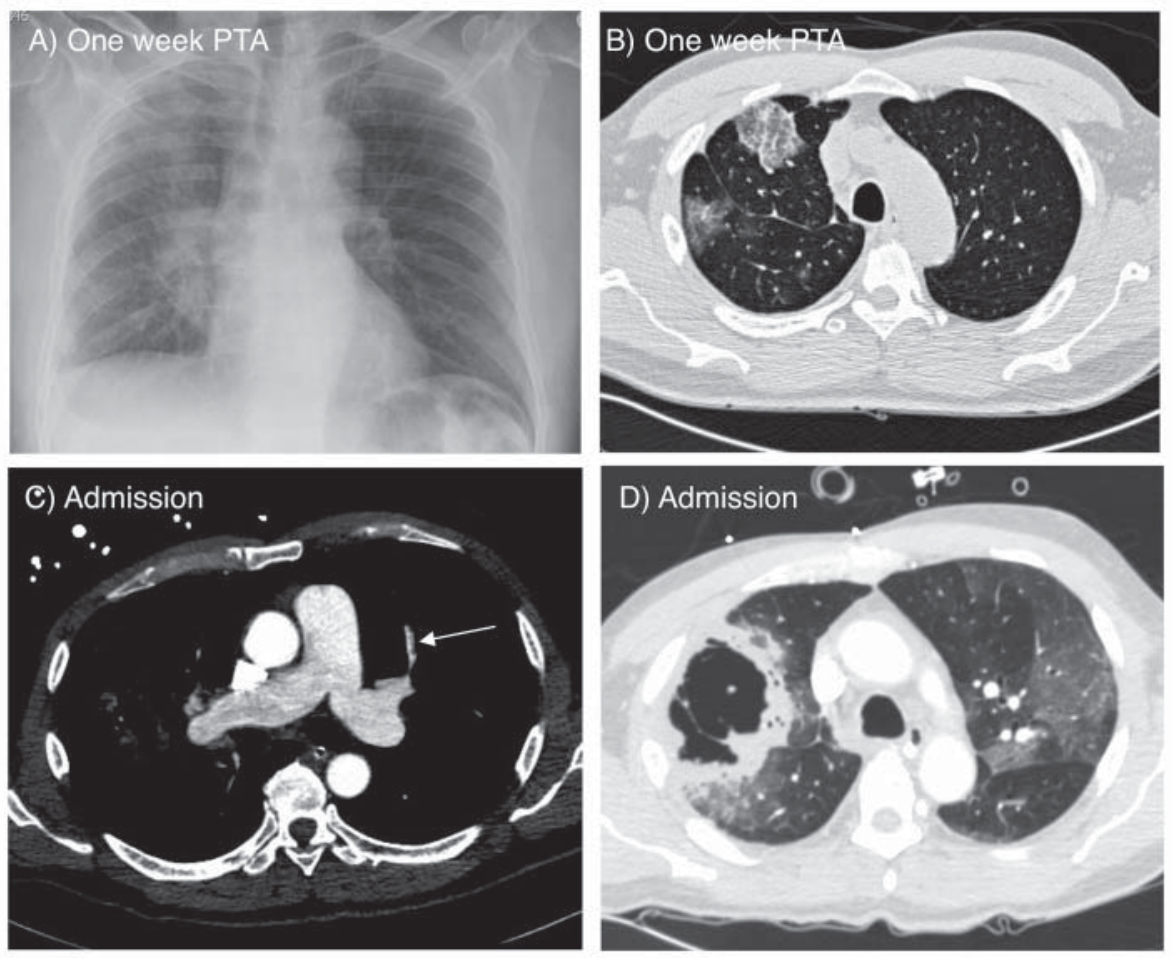

Figure 1. A) Chest radiograph on admission showing ground glass opacity and infiltrate in the right superior lung field and right perihilar area. B) Axial CT scan of the chest obtained one week prior to admission showing ground glass opacifications in the right middle and lower lobes. C) CT angiogram on admission showing a small embolus in a pulmonary artery branch of the left upper lobe (arrow). D) Lung window image of the CT angiogram demonstrating two adjacent large thick wall cavities in the right middle and lower lobes, corresponding to the ground glass opacities seen one week prior. There are also bilateral ground glass opacities.

of patients [1]. Most reported cases of TE are associated with breast, lung, or gastric adenocarcinomas, the same tumor types that put individuals at increased risk for VTE [1, 2]. Discerning TE from non-malignant VTE is further complicated because VTE is approximately twice as common in patients with malignancy as in those without [3].

The utility of advanced imaging for revealing TE is largely unknown. Most cases of documented TE to the lungs have not shown any significant parenchymal abnormalities on contrast-enhanced computed tomography (CT) of the chest although "dilated and beaded" subsegmental pulmonary arteries have been described [1, 2]. Ventilationperfusion scintigraphy can reveal multiple peripheral and subsegmental mismatched defects [1]. Short of a lung biopsy, cytologic analysis of a blood sample obtained from the distal port of a wedged pulmonary artery catheter is a definitive way of making the diagnosis although the sensitivity of this maneuver is not known [1]. Cavitary lung lesions are most often associated with infectious processes, but rapidlydeveloping cavitations can occur in the setting of infarction due to VTE or necrosis secondary to a malignancy [4-6].

We present the case of rapidly-forming cavitary lung lesions due to pulmonary infarction from diffuse TE and is, to the best of our knowledge, the first such case reported.

\section{Case Report}

\section{History of present illness}

A 64 year-old man who was diagnosed two years ago with Stage 2a (T1N1) adenocarcinoma of the lung presented with acute onset of shortness of breath. Treatment for his cancer included a right upper lobectomy and adjuvant chemotherapy with carboplatin and taxol. Surgical margins of the resected lobe were clear of malignancy and follow-up surveillance imaging had not revealed any clear evidence of recurrence. A persistent non-productive cough began six weeks prior to admission (PTA). Chest radiograph obtained one week PTA revealed vague patchy infiltrate in the right superior lung field and right perihilar areas (Fig. 1A). Noncontrasted chest CT obtained at the same time demonstrated only ground glass opacities in the right middle and lower lobes (Fig. 1B). Bronchoscopy at that time revealed scant hemorrhage from the right lower lobe. All cultures and cytology from bronchoalveolar lavage fluid were negative.

\section{Physical exam and data}

On presentation, temperature was $99.2{ }^{\circ} \mathrm{F}$, heart rate 120 


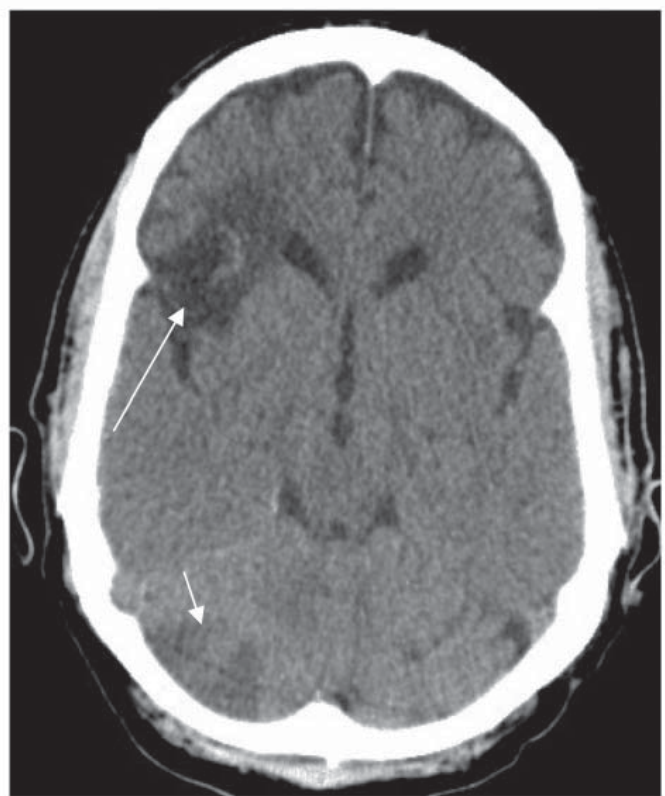

Figure 2. CT of the head showing hypodense lesions in the right frontal (long arrow) and cerebellar lobes (short arrow) consistent with embolic infarctions.

beats per minute, blood pressure 114/72 $\mathrm{mmHg}$, respiratory rate 32 breaths per minute, and oxygen saturation of $84 \%$ on room air. Notable findings included diminished breath sounds in both lung bases, and crackles over the right superior lung field.

White blood cell count was $21500 / \mathrm{mL}$ with $88.5 \%$ neutrophils. Hemoglobin concentration was $14.4 \mathrm{~g} / \mathrm{dL}$. Serum lactate concentration was $4 \mathrm{mmol} / \mathrm{L}$. Troponin level was 0.07 $\mathrm{ng} / \mathrm{mL}$ and reached a peak of $0.53 \mathrm{ng} / \mathrm{mL}$. Arterial blood gas demonstrated a $\mathrm{pH}$ of $7.49, \mathrm{PaCO}_{2}$ of $22.9 \mathrm{mmHg}, \mathrm{PaO}_{2}$ of $67 \mathrm{mmHg}$, and oxygen saturation of $95 \%$ on $4 \mathrm{~L} /$ minute of supplemental oxygen. Liver tests were notable for an aspartate aminotransferase of $96 \mathrm{IU} / \mathrm{L}$, alanine aminotransferase of $101 \mathrm{IU} / \mathrm{L}$, and an alkaline phosphatase of $414 \mathrm{IU} / \mathrm{L}$. Anti- neutrophil cytoplasmic antibody (ANCA) and anti-nuclear antibody (ANA) tests were negative.

CT angiogram on admission revealed a pulmonary embolus to the left upper lobe (Fig. 1C). Two large, thick-walled cavities in the right middle and lower lobes, bilateral ground glass opacities, and a small right pleural effusion were also seen (Fig. 1D). The locations of the cavities corresponded to the ground glass opacities seen on the CT obtained only one week prior (Fig. 1B).

\section{Hospital Course}

The patient was begun on intravenous heparin for venous thromboembolism (VTE) as well as broad-spectrum antibiotics for possible necrotizing bacterial pneumonia. Echocardiogram demonstrated normal left ventricular function but right ventricular systolic dysfunction with septal flattening consistent with elevated right ventricular pressure. Pulmonary arterial systolic pressure was estimated to be $37 \mathrm{mmHg}$ but may have been under-estimated due to poor tricuspid articulation.

On the second hospital day, increasing oxygen requirement and markedly-increased labor of breathing necessitated endotracheal intubation and mechanical ventilation. All sputum and blood cultures were negative for bacterial pathogens. On the seventh hospital day, he became obtunded despite adequate oxygenation and blood pressure. Noncontrast CT of the brain revealed multiple hypodense areas with lesions in the right frontal lobe and right cerebellar lobe (Fig. 2). Given the grave prognosis, comfort care was instituted after family members conveyed his wishes to the medical team. The patient died shortly thereafter.

\section{Post-mortem Analysis}

Post-mortem examination was restricted to the lungs and liver. Sectioning of the lungs did not demonstrate any gross VTE in the main pulmonary arterial branches. Examination

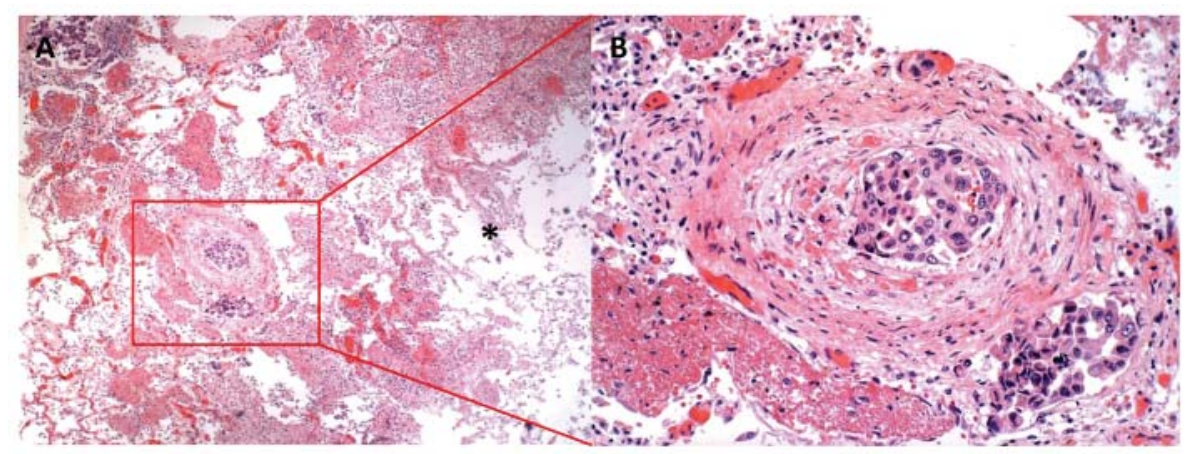

Figure 3. Lung histopathology showing extensive tumor emboli. A) Low power image of right middle lobe with tumor embolus showing congested, but viable tissue to the left of the embolus and necrotic cavitary abscess to the right $\left({ }^{*}\right)(40 \mathrm{X})$. B) Higher power image of arterial and lymphatic/venous tumor emboli adhering to the endothelium and obstructing the vascular luminae $(200 \mathrm{X})$. 
of hematoxylin and eosin stained lung tissues demonstrated innumerable TE throughout both lungs including prominent TE in vessels leading to the cavitary lesions (Fig. 3). Cell morphology and focally-positive staining for mucin and thyroid transcription factor-1 (TTF-1) was consistent with lung adenocarcinoma. Acid-fast and Grocott's methenamine silver (GMS) stains of the lung tissues around the cavities were negative for acid-fast organisms or fungi. There were two foci of intrapulmonary metastases, one in the left lower lobe and one in the right lower lobe. Neither of these metastatic foci showed significant tumor necrosis and the right lower lobe focus was separate from the cavitary lesions of the right lung. TE also invaded the splanchnic circulation with widespread hepatic involvement, causing portal vein thrombosis and parenchymal metastasis causing centrilobular necrosis. Although post-mortem examination was not performed on the brain, cerebral TE with brain infarctions were likely responsible for the obtundation and the abnormal brain imaging.

\section{Discussion}

This case describes a patient with rapidly developing cavitary lung lesions associated with respiratory failure. It illustrates that in someone with a history of a malignancy, this unusual complication of cancer (i.e., TE), which can mimic other disorders and is difficult to diagnose pre-mortem, should be considered in the differential diagnosis. Since our patient did not have evidence of VTE to the right lung on CT angiogram or at autopsy, and there was no evidence of an infectious etiology for his rapid deterioration, we posit that embolic infarction from widespread TE caused the rapidly developing cavities; this complication of TE, to the best of our knowledge, has not been previously reported.

The overall prognosis of critically ill patients with TE is grave, despite treatment with chemotherapy [1]. Because the diagnosis of TE is frequently not considered and is difficult to confirm pre-mortem, the diagnosis is often revealed at autopsy $[1,2]$. Both TE and VTE may simultaneously occur in the pulmonary capillary beds [1]; we believe that in our patient, however, TE was the cause of the lung cavities and respiratory failure. Additionally, the presence of TE in the lung likely indicates diffuse involvement elsewhere in the body $[2,7]$. Because microthrombi may simultaneously occur with TE, optimal treatment may require therapy for both types of clot burden with anticoagulation as well as chemotherapy [2]. Treating both processes early and aggressively may prolong survival [8].
In summary, we propose that extensive TE can rarely cause cavitary infarction of the lungs and should be considered in the differential diagnosis of rapidly-developing cavities, particularly in patients with a known history of malignancy. Early recognition of atypical manifestations of TE may allow more effective therapies and ultimately improve outcomes.

\section{Grant Support}

None.

\section{Conflicts of Interest}

None.

\section{References}

1. Roberts KE, Hamele-Bena D, Saqi A, Stein CA, Cole RP. Pulmonary tumor embolism: a review of the literature. Am J Med. 2003;115(3):228-232.

2. Liang YH, Kuo SW, Lin YL, Chang YL. Disseminated microvascular pulmonary tumor embolism from nonsmall cell lung cancer leading to pulmonary hypertension followed by sudden cardiac arrest. Lung Cancer. 2011;72(1):132-135.

3. Stein PD, Beemath A, Meyers FA, Skaf E, Sanchez $\mathrm{J}$, Olson RE. Incidence of venous thromboembolism in patients hospitalized with cancer. Am J Med. 2006;119(1):60-68.

4. Libby LS, King TE, LaForce FM, Schwarz MI. Pulmonary cavitation following pulmonary infarction. Medicine (Baltimore). 1985;64(5):342-348.

5. Gadkowski LB, Stout JE. Cavitary pulmonary disease. Clin Microbiol Rev. 2008;21(2):305-333, table of contents.

6. Kolodziejski LS, Dyczek S, Duda K, Goralczyk J, Wysocki WM, Lobaziewicz W. Cavitated tumor as a clinical subentity in squamous cell lung cancer patients. Neoplasma. 2003;50(1):66-73.

7. Nakano M, Miwa K, Kanimoto Y, Ishihara S, Deguchi T. Microscopic pulmonary tumor embolism secondary to adenocarcinoma of the prostate. Hinyokika Kiyo. 2003;49(3):169-172.

8. Shao L, Wang ZH, Zuo JY. Clinical analysis of 23 lung cancer patients accompanied by pulmonary embolism. Chinese J Cancer 2009; 28:1-5. 